VOL. 18 (1978), 95-98.

\title{
A functorial version of a construction of Hochschild and Mostow for representations of Lie algebras
}

\section{William H. Wilson}

Let $\underline{\mathrm{g}}$ be a lie algebra, $\underline{\underline{\mathrm{h}}}$ a complemented ideal of $\underline{\mathrm{g}}$, and $W$ an $\underline{\underline{h}}$-module. Hochschild and Mostow have described the construction of a g-module "induced" from $W$, which is finitedimensional provided $W$ is finite-dimensional and satisfies a nilpotent action condition. This note describes a modification of their construction which is functorial and a weak adjoint to the restriction functor from g-modules to $\underline{\underline{h}}$-modules.

Throughout this paper we shall suppose that $g$ is a Lie algebra over a field $k$, that $\underline{\underline{h}}$ is an ideal of $\underline{\underline{B}}$, and that there is a subalgebra $\underline{\underline{S}}$ of $\underline{g}$ such that $\underline{\underline{g}}=\underline{\underline{h}} \oplus \underline{\underline{s}}$. U U algebras of $\underline{\underline{h}}$ and $\underline{\underline{g}}$. Clearly, every $g \in \underline{\underline{g}}$ can be written uniquely as $g=h+s$ with $h \in \underline{\underline{h}}$ and $s \in \underline{s}$. This allows us to define, (with Hochschild and Mostow [1]), a composition * by

$$
g * u=h u+(s u-u s) \text { for } g \in \underline{g}, u \in U \underline{\underline{h}} \text {. }
$$

It can be shown that $s u-u s \in U \underline{\underline{h}}$, hence (1) determines a $\underline{\underline{g}}$-module structure on $U \underline{\underline{h}}$.

We shall use mod- $\underline{\underline{h}}$, mod- $\underline{\underline{g}}$ to denote the categories of right $\underline{\underline{h}-}$ and g-modules, and $F: \bmod -\underline{\underline{g}} \rightarrow \bmod -\underline{\underline{h}}$ to denote the restriction functor. Now let $W \in \bmod -\underline{\underline{h}}$. Then $\operatorname{hom}_{k}(\underline{\underline{h}}, W)$ has a g-module structure given by

Received 8 November 1977. The material of this paper formed part of the author's $\mathrm{PhD}$ thesis, which was written under the supervision of Dr D.W. Barnes, to whom the author wishes to express his thanks. 
$f^{\theta}(u)=f(g * u)$ for $f \in \operatorname{hom}_{k}(\underline{\underline{W h}}, W), g \in \underline{\underline{g}}$, and $u \in U \underline{\underline{\mathrm{h}}}$.

\section{Construction of the functor $I$}

Define a map $\hat{j}_{W}: W \rightarrow \operatorname{hom}_{k}(U \underline{\underline{h}}, W)$ by setting $\hat{j}_{W}(w)(u)=w \cdot u$ for

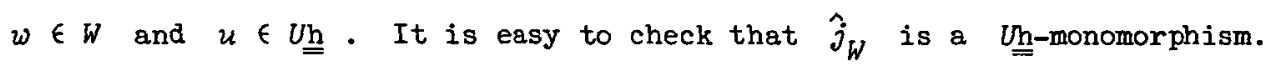
We define a g-submodule $T W$ of $\operatorname{hom}_{k}(\underline{\underline{h}}, W)$ by setting $I W=\left(\operatorname{im} \hat{j}_{W}\right) \cdot U_{\underline{\underline{B}}}$. Now let $j_{W}$ be $\hat{j}_{W}$ with codomain restricted to be $F I W$. Let $W, W^{\prime} \in \bmod \underline{\underline{h}}$ and $\psi \in \operatorname{hom}_{\underline{\underline{h}}}\left(W, W^{\prime}\right)$. We define $I \psi: I W \rightarrow I W^{\prime}$ by $[(I \psi)(f)](u)=(\psi \circ f)(u)$ for $u \in U \underline{\underline{h}}$ and $f \in I W$. We must show $(I \psi)(f) \in I W^{\prime}$. Since $f \in I W, f$ may be written as

$$
f=\sum_{i=1}^{n} j_{W}\left(w_{i}\right) \cdot x_{i}
$$

for suitable $w_{i} \in W, x_{i} \in \underline{\underline{g}}$. Then, for $u \in U \underline{\underline{h}}$,

$$
\begin{aligned}
{[(I \psi)(f)](u) } & =\left[\psi \circ \sum_{i=1}^{n} j_{W}\left(w_{i}\right) \cdot x_{i}\right](u) \\
& =\psi\left(\sum_{i=1}^{n} x_{i}\left(x_{i} * u\right)\right) \\
& =\sum_{i=1}^{n} \psi\left(w_{i}\right) \cdot\left(x_{i} * u\right) \\
& =\left[\sum_{i=1}^{n} j_{W^{\prime}}\left(\psi\left(w_{i}\right)\right) \cdot x_{i}\right](u),
\end{aligned}
$$

so $(I \psi)(f)=\sum_{i=1}^{n} j_{W^{\prime}}\left(\psi\left(w_{i}\right)\right) x_{i} \in I W^{\prime}$. If $W^{\prime \prime}$ is another h-module and $\psi^{\prime} \in \operatorname{hom}_{U \underline{\underline{h}}}\left(W^{\prime}, W^{\prime \prime}\right)$, then $\left(\psi^{\prime} \circ \psi\right) \circ f=\psi^{\prime} \circ(\psi \circ f)$; it follows that $I$ has the multiplicative property of a functor.

LEMMA 1. $j_{W}$ is natural in $W$.

Proof. We must show that if $\psi \in$ hom $_{U h}\left(W, W^{\prime}\right)$ then $F I \psi \circ j_{W}=j_{W^{\prime}} \circ \psi$. Suppose $\omega \in W$ and $u \in U \underline{\underline{\underline{h}}}$. Then 
$\left(E I \psi \circ j_{W}\right)(w)(u)=\left(\psi \circ j_{W}(w)\right)(u)=\psi(w \cdot u)=\psi(w) \cdot u=j_{W^{\prime}}(\psi(w))(u)$

$$
=\left(j_{W}, \circ \psi\right)(w)(u)
$$

as required.

LEMA 2. I is a faithful functor.

Proof. If $I \psi=0$, then for all $w \in W, 0=(I \psi)\left(j_{W}(w)\right)$; so $0=\left[(I \psi)\left(j_{W}(w)\right)\right]\left(I_{\underline{\underline{\underline{h}}}}\right)=\psi(w)$. That is, $\psi=0$.

THEOREM 3. The functor $I: \bmod -\underline{\underline{h}} \rightarrow \bmod -\underline{\underline{g}}$, described above, is an injective weak left adjoint to $F$. That is, for $W \in \bmod -\underline{\underline{h}}$ and $V \in \bmod -\mathrm{g}$, there is an injection

$$
\theta_{W V}: \operatorname{hom}_{U \underline{\underline{g}}}(I W, V) \rightarrow \operatorname{hom}_{U \underline{\underline{h}}}(W, F V)
$$

which is natural in $W$ and $V$.

Proof. For $\phi \in \operatorname{hom}_{U_{\underline{\underline{g}}}}(I W, V)$, we define $\theta_{W V}(\phi)=F \phi \circ j_{W}$. The naturality of $\theta_{W V}$ follows from that of $j_{W}$ and the definition of $\theta_{W V}$. We must show that $\theta_{W V}$ is injective. Suppose that $\phi_{1}, \phi_{2} \in$ hom $_{U_{\underline{\underline{g}}}}(I W, V)$, and that $F \phi_{1} \circ j_{W}=F \phi_{2} \circ j_{W}$. Then $\phi_{1}$ and $\phi_{2}$ coincide on im $j_{W}$.

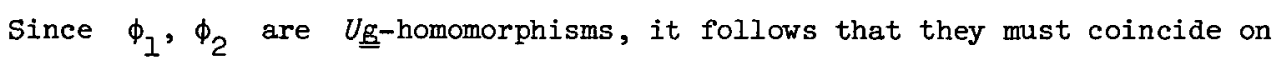
$\left(\right.$ im $\left.j_{W}\right) \cdot U_{\underline{g}}=I W$.

THEOREM 4 (compare Hochschild and Mostow [1] and Zassenhaus [2]). Let $\underline{\underline{g}}$ be a finite-dimensional Lie algebra over a field $k$ of characteristic zero, and let $\underline{\underline{h}}$ be an ideal of $\mathrm{g}$ with complementary

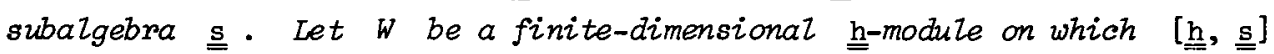
acts nilpotently. Then IW, as defined above, is a finite-dimensional g-module.

Proof. If $\{0\}=W_{0}<W_{1}<\ldots<W_{n}=W$ is a composition series for $W$, then set $S(W)=\left(W_{n} / W_{n-1}\right) \oplus \ldots \oplus\left(W_{2} / W_{1}\right) \oplus\left(W_{1} / W_{0}\right)$. By the JordanHölder theorem, $S(W)$ is determined up to isomorphism. Clearly, a subalgebra of $\underline{\underline{h}}$ acts nilpotently on $W$ if and only if it annihilates $S(W)$. Let us write $d=\operatorname{dim}_{k} W$, and let $\operatorname{ann}_{U_{\underline{h}}}(M)$ denote the annihilator in $U \underline{\underline{h}}$ of an $\underline{\underline{h}-m o d u l e ~} M$. Obviously, 


$$
\left(\operatorname{ann}_{U \underline{\underline{h}}}(S(W))\right)^{d} \subseteq \operatorname{ann}_{U \underline{\underline{\underline{h}}}}(W) \subseteq \operatorname{ann}_{U_{\underline{\underline{h}}}}(S(W))
$$

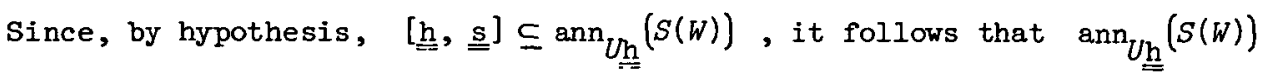
is a g-submodule of $W$. Hence $\left(a n_{U \underline{\underline{h}}}(S(W))\right)^{d}$ is a g-submodule of $\underline{\underline{\underline{h}}}$

$$
\begin{aligned}
& \text { If } \begin{array}{r}
f \in \operatorname{hom}_{k}(U \underline{\underline{h}}, W) \text { and } f\left(\operatorname{ann}_{U \underline{\underline{h}}}(W)\right)=\{0\} \text {, then for } \\
f^{x}\left\{\left(\operatorname{ann}_{\underline{\underline{\underline{h}}}}(S(W))\right)^{d}\right\} \subseteq f\left(x \cdot\left(\operatorname{ann}_{\underline{\underline{U_{h}}}}(S(W))\right)^{d}\right\}
\end{array} \\
& \subseteq f\left(\mathrm{ann}_{U \underline{\underline{\mathrm{h}}}}(W)\right) \text { by (2), } \\
& =\{0\} \text {. }
\end{aligned}
$$

Now im $j_{W}$ annihilates $\operatorname{ann}_{\underline{\underline{U_{h}}}}(W) ;$ so $I W=\left(\right.$ im $\left.j_{W}\right) \cdot U_{\underline{\underline{g}}}$ annihilates $\left(\operatorname{ann}_{U \underline{\underline{h}}}(S(W))\right)^{d}$. Let us write $J=\left(\operatorname{ann}_{U \underline{\underline{\underline{h}}}}(S(W))\right)^{d}$. Then it is easy to see that $I W$ is embedded in $\operatorname{hom}_{k}(U \underline{\underline{h}} / J, W)$. Since $W$ is finitedimensional, $\operatorname{ann}_{U \underline{\underline{h}}}(W)$ is of finite codimension in $U \underline{=}$. Hence, by (2),

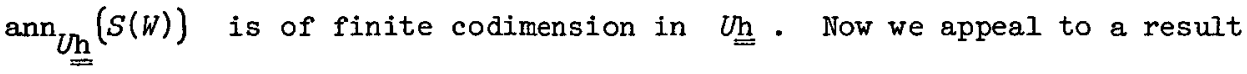
of Zassenhaus [2, page 263], which states that if $X, Y$ are ideals of $\underline{\underline{W h}}$ of finite codimension, then so is $X Y$. We deduce from this that $J$ is of finite codimension in $\underline{\underline{h}}$, so that $\operatorname{dim}_{k} \operatorname{hom}_{k}(\underline{\underline{h}} / J, W)<\infty$, and so $\operatorname{dim}_{k} I W<\infty$.

\section{References}

[1] G. Hochschild and G.D. Mostow, "Extensions of representations of Lie groups and Lie algebras, I", Amer. J. Math. 79 (1957), 924-942.

[2] Hans Zassenhaus, "Über die Darstellungen der Lie-Algebren bei Charakteristik 0 ", Comment. Math. Helv. 26 (1952), 252-274.

Department of Mathematics, University of Queensland, St Lucia, Queensland. 